\section{Generic prescribing and the drug industry}

Britain's NHS general practitioners have always valued their freedom to prescribe whatever drugs they think best for their patients; this right is, they claim, an essential feature of professional, clinical independence. Many of their hospital colleagues have much less freedom: their prescribing is constrained by hospital drug formularies and antibiotic policies agreed voluntarily to cut costs and improve standards of drug treatment. Many other countries (the Netherlands and Australia, for example) limit the range of drugs prescribable within the State health-care system. Yet general practitioners in Britain remain free to prescribe the latest (and often the most expensive) non-steroidal anti-inflammatory drug, antidepressive, or hypnotic and to prescribe vitamins as placebos or "tonics."

So no one can have been surprised that the Conservative Government, elected on a commitment to cut costs, should have set up a working group on effective prescribing with members drawn from the British Medical Association, its General Medical Services Committee, the Royal College of General Practitioners, the Joint Consultants Committee, and the Department of Health and Social Security.

As is so often the case nowadays, the working group's report has been leaked ${ }^{1}$ but not yet published (though it is said to have been sent to the Secretary of State for Social Services in mid-February). The leaks suggest that the report includes a recommendation that when prescriptions by general practitioners name a branded product the pharmacist should be able to supply a cheaper generic alternative unless specifically instructed not to do so. Such a recommendation would be in tune with those current public attitudes that see pharmaceutical companies as making vast profits and believe that drugs are overpriced.

Predictably, the Association of the British Pharmaceutical Industry has mounted a campaign against generics. ${ }^{2}$ Firstly, it claims that the savings would be small when set against NHS expenditure and would amount to only $0.3 \%$ of the total or around $f 24 \mathrm{~m}$ a year in 1980 . Secondly, the Association of the British Pharmaceutical Industry believes that if Britain legalises generic substitution other countries would follow suit with disastrous effects on major drug companies' research programmes. Thirdly, generic substitutes are said sometimes to differ substantially from branded products in their bioavailability and their consistency.

The last of these three objections is, perhaps, the most substantial. In the context of doctors' clinical responsibilities the variable quality of generics is worrying: those manufactured by the major companies may be satisfactory, but some imports from Eastern Europe and the Far East do not inspire the same confidence. In the United States, where cost-conscious consumers have pushed generics' share of the total drug market to $25 \%{ }^{3}$ a law suit is already pending in which a patient alleges damage from a generic equivalent supplied by a pharmacist. ${ }^{4}$ Where would liability lie for alleged damage of this kind if the NHS introduced generic substitution?

In practical terms, the NHS negotiates the prices paid for drugs with their manufacturers through the Pharmaceutical Price Regulation Scheme. This relates the total earnings of the industry to investment in pharmaceutical research in Britain and should ensure that both sides get a reasonably fair deal. If the Government did introduce generic substitution this balance would be disturbed and the industry would be affronted. At present the British pharmaceutical industry is a major source of overseas earnings ( $£ 800 \mathrm{~m}$ a year) and it employs 70000 people. $^{2}$ Multinational companies can, however, direct their investment where they wish and they look with little enthusiasm at countries, such as Australia, which drive too hard a bargain on health service drugs.

Other ways can surely be found to make prescribing more economical. Provided patent life is properly protected there might, for example, be a case for asking patients to pay a proportion of the cost of drugs prescribed within 12 months of marketing - and so avoid repetitions of recent episodes of clamour for the latest product inspired by promotion aimed directly at the public. When and if the working group's recommendations are published the options can be discussed and analysed. We believe, however, that generic substitution would be a clumsy and ineffective way of reducing NHS drug costs and one which might discourage foreign investment in the British pharmaceutical industry. Long term, a worldwide trend towards use of generics could further reduce the pace and breadth of research by the pharmaceutical industry.

1 Cowhig J. Doctors propose plan for more generic drugs. General Practitioner 1982 March 5:12-5.

${ }^{2}$ Association of the British Pharmaceutical Industry. The importance of brand names. Pharmatopics $1982 ; 3: 1-8$.

3 Barrie GJ. The marketing of generic drugs. London: Associated Business Press, 1981.

${ }^{4}$ Dickinson J. First substitution-linked death raises major issues. Pharmaceutical fournal $1981 ; 227: 482$.

\section{Thin students for thin medical schools}

Not only are Britain's medical schools heading for a thin time (30 January, p 335), but so also are their students. The Government is proposing to increase student grants for the next academic year by only $4 \%$, which at current inflation rates will mean a real cut of about $8 \%$. All students will suffer, but clinical medical students, who work a 48-week year, will be hit especially hard. Special pleading is not to be undertaken lightly, but are the bureaucrats who cut with such bravado aware that because of unavoidable conditions some groups will suffer more than others?

Clinical students receive the same grant, $£ 60$ a week, as all other students for the normal 30-week year. But while other students can head for a summer grape-picking in France or living cheaply in Goa, clinical students must soldier on for another 18 weeks-only now on $£ 37.50$ a week. Their expenses are certainly no less than those of other students (indeed, with the high standard of dress expected and the high cost of medical textbooks they are probably higher), and if they give their medicine the attention it should have they have no time to work in bars or holiday-camps. A recent survey carried out by the students' union at Charing Cross Hospital showed that students spent on average $£ 2421$ of their allowed $£ 2650$ on essentials-food, accommodation, etc. They were left $£ 4.40$ a week for recreation -one cinema visit to be washed down with one pint of beer at London prices. The real cut of $8 \%$ will remove that taste of luxury.

Furthermore, cuts of this kind will affect not only individual students but also eventually the practice of medicine in Britain. Hard times might make for good poets, but there is no evidence that they make for better doctors. On the contrary, if students neglect the wards for part-time work, standards are 